\title{
Optical activity of catalytic elements of hetero-metallic nanostructures
}

\author{
Tomasz J. Antosiewicz, ${ }^{a, b, \dagger}$ S. Peter Apell ${ }^{b}$, Carl Wadell ${ }^{b}$, and Christoph Langhammer, ${ }^{b}$ \\ ${ }^{a}$ Centre of New Technologies, University of Warsaw, Banacha 2c, 02-097 Warsaw, Poland; \\ ${ }^{b}$ Department of Applied Physics, Chalmers University of Technology, \\ SE-412 96 Göteborg, Sweden
}

\begin{abstract}
Interaction of light with metals in the form of surface plasmons is used in a wide range of applications in which the scattering decay channel is important. The absorption channel is usually thought of as unwanted and detrimental to the efficiency of the device. This is true in many applications, however, recent studies have shown that maximization of the decay channel of surface plasmons has potentially significant uses. One of these is the creation of electron-hole pairs or hot electrons which can be used for e.g. catalysis. Here, we study the optical properties of hetero-metallic nanostructures that enhance light interaction with the catalytic elements of the nanostructures. A hybridized LSPR that matches the spectral characteristic of the light source is excited. This LSPR through coupling between the plasmonic elements maximizes light absorption in the catalytic part of the nanostructure. Numerically calculated visible light absorption in the catalytic nanoparticles is enhanced 12 -fold for large catalytic disks and by more 30 for small nanoparticles on the order of $5 \mathrm{~nm}$. In experiments we measure a sizable increase in the absorption cross section when small palladium nanoparticles are coupled to a large silver resonator. These observations suggest that heterometallic nanostructures can enhance catalytic reaction rates.
\end{abstract}

Keywords: plasmonics, catalysis, transition metals, solar harvesting, plasmon-assisted photocatalysis

\section{INTRODUCTION}

Interaction of light with metals in the form of surface plasmons is used in a wide range of applications, such as biosensing, ${ }^{1-3}$ photodetection, ${ }^{4,5}$ waveguiding, ${ }^{6,7}$ light emission ${ }^{8,9}$ and materials science. ${ }^{10}$ These applications require that the scattered component (alternatively, transmission) is maximized in order to achieve superior working parameters. The absorption decay channel is in this context thought of as parasitic and usually every effort is made to minimize it. However, recent work has shown that the absorption decay channel of surface plasmons has interesting and potentially significant uses. For example, it has been demonstrated that localized surface plasmons can excite hot electrons in metals, ${ }^{11-13}$ which can later be utilized in a number of ways. One of the most exciting ones is plasmon-assisted photocatalysis. ${ }^{14}$ Plasmon-assisted photocatalysis on noble metals has the benefit of providing hot electrons via the localized surface plasmon resonance (LSPR) and simultaneously the catalytically active site. ${ }^{15}$ However, noble metals (narrowly defined as the classic plasmonic metals $\mathrm{Au}$ and $\mathrm{Ag}$ ) are limited in terms of their catalytic properties and other materials, such as transition metals, offer a wider range of reactions catalyzed by their surfaces. ${ }^{16}$ Unfortunately, transition metals exhibit rather poor plasmonic properties (low field enhancements, low resonance quality factors) and additionally exhibit LSPRs only in the UV for nanometer-sized particles. ${ }^{17}$ This mismatch between the LSPR and the solar maximum implies low solar harvesting efficiencies. A natural solution to this problem is to shift (enhance) the plasmonic activity of poor metals into the visible.

Here, we describe recent results of optical properties of hetero-metallic nanostructures designed in such a way as to enhance light interaction with the catalytic elements of the nanostructures. We excite a hybridized LSPR that matches the spectral characteristic of the light source and at the same time maximizes light absorption in the catalytic metal element of the nanostructure. We show that visible light absorption in the catalytic

\footnotetext{
${ }^{\dagger}$ tomasz.antosiewicz@uw.edu.pl
} 


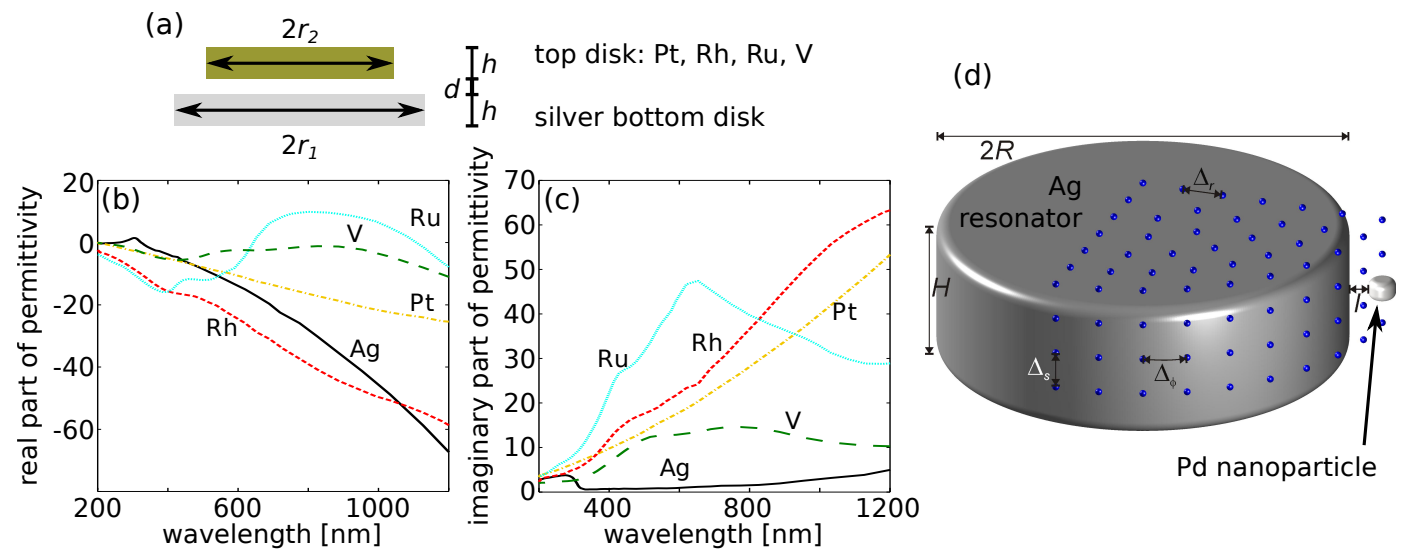

Figure 1. (a) Model nanostructure with a catalytic metal top disk and a silver bottom disk. (b,c) Real and imaginary parts of permittvities of the catalytic metals used in calculations shown in (a). (d) Scheme of the nanostructure composed of a large silver nanoantenna coupled to a tiny palladium nanoparticle.

nanoparticles can be enhanced by more than one order of magnitude, depending on the metal. However, such model systems with large catalytic particles are not feasible, since the available surface-to-volume ratio is not optimum. Catalysis is much more efficient when an active material is dispersed in the form of nanometersized particles. Thus, in the second part of our work we investigate coupling between a large silver disk and random arrangements of nanometer-sized palladium nanoparticles. For a random array of Pd nanoparticles the average enhancement is on the order of 10 , while the maximum exceeds 30 . These effects of enhanced absorption imply that whenever the decay channel in a catalytic material leads to generation of hot electrons or electron-hole pairs, then by coupling this material with good plasmonic resonators it is possible to enhance absorption in the catalyst. Ultimately, this may lead to enhanced catalytic reaction rates for light absorption limited processes.

\section{METHODS}

\subsection{Numerical calculations}

All numerical calculations are carried out using the finite-difference time-domain (FDTD) method with a commercial code implemented by Lumerical, Inc., Canada. The good resonator, whose role it to collect efficiently incident light, is made of silver and has a varied radius $r_{1}$ from 50 to $80 \mathrm{~nm}$. The reason for varying its radius is to scan the LSPR across the peak of the solar spectrum. In the first case when both disks are large (Fig. 1a) the top disk is made of either $\mathrm{Pt}, \mathrm{Rh}, \mathrm{Ru}$, and $\mathrm{V}$, which are all good catalytic materials, but poor plasmonic metals. Both disks have the same thickness $h=20 \mathrm{~nm}$ and the second one has a variable radius $r_{2}$ from $10 \mathrm{~nm}$ to the size of the bottom $\mathrm{Ag}$ disk. We vary the separation distance $d$ to see how the absorption enhancement is affected by the strength of the coupling. $d=0,10$, and $20 \mathrm{~nm}$. The aim is to discover how different material properties (permittivity) affect coupling and absorption.

The second part of the study is conducted for one large disk that is surrounded by one (in calculations) or more (in experiments, details to follow in next section) palladium nanoparticles. The $\mathrm{Ag}$ nanoantenna for coupling light into the $\mathrm{Pd}$ has a variable radius $R$ from 30 to $90 \mathrm{~nm}$ and its thickness is $20 \mathrm{~nm}$. The $\mathrm{Pd}$ nanoparticle is in the shape of a disk $4 \mathrm{~nm}$ in diameter and $3 \mathrm{~nm}$ in height - its size is based on TEM images of Pd nanoparticles fabricated for sintering studies. ${ }^{18}$ We move the Pd nanoparticle around the $\mathrm{Ag}$ disk to probe the spatial response to the presence of the catalytic material. The probing positions are obtained by first dividing the cross section normal to the base of the disk into equidistant segments whose length along the base is $\Delta_{r}$ and along the side $\Delta_{s}$ - see Fig. 1d for details. Each point chosen this way defines a circle with a certain radius. These circles are then further divided into azimuthal segments $\Delta_{\phi}$ in length. Due to symmetry we only need to calculate one-eighth of the total points. For the $R=30 \mathrm{~nm}$ disk we run 70 calculations (corresponding to $\frac{1}{8}$ of all points) and for 140 for the largest $90 \mathrm{~nm}$ disk. This way we obtain an array of positions which denote positions at which we place the Pd nanoparticle for absorption enhancement 
studies. In a real catalytic process the Ag disk would have to be protected from the harsh reactive environment by a thin spacer. We mimic this by setting a separation between the $\mathrm{Pd}$ and $\mathrm{Al}$ to $l=5 \mathrm{~nm}$.

FDTD calculations are done with total-field/scattered-field linearly polarized excitation and absorbing boundary conditions. We use a nonuniform mesh with a minimum step of $1 \mathrm{~nm}$ around the large disks and $8 \mathrm{~nm}$ far away from them with a graded transition region. The palladium nanoparticles are meshed with a step of $0.5 \mathrm{~nm}$. The time step is calculated from Courant's stability criterion. All permittivities are taken from literature. ${ }^{19}$ Figure 1b,c presents the real and imaginary parts of permittivity of the metals used in the multi-metal study of the first part.

Absorption enhancement is calculated by evaluating the product of the imaginary part of the permittivity of the material in question and the field intensity present in its volume. We calculate wavelength-dependent absorption enhancement $A_{\lambda}$, which is defined as the ratio of absorption in the catalytic element of the nanostructure to absorption in the same catalytic particle placed in vacuum, and wavelength-integrated (total) absorption enhancement according to

$$
A_{t}=\frac{\int \sigma_{a}^{*}(\lambda) s(\lambda) d \lambda}{\int \sigma_{a}(\lambda) s(\lambda) d \lambda}
$$

where the integrations run over the spectral range used in our calculations, i.e. from 280 to $1200 \mathrm{~nm}$. Here $\sigma_{a}^{*}(\lambda)$ and $\sigma_{a}(\lambda)$ are the absorption cross sections of coupled and uncoupled catalytic particles, respectively, and $s(\lambda)$ is the spectrum of the light source, in our case the solar spectrum.

\subsection{Experimental methods}

The samples are fabricated on borosilicate glass substrates. Amorphous arrays of Ag nanodisks are made using hole-mask colloidal lithography, ${ }^{20}$ which utilizes self-assembly of charged polystyrene nanobeads to produce an evaporation mask. Silver is evaporated onto the substrate through this mask using physical vapor deposition. The morphologies of the Ag disks and the array as a whole are dictated by the polystyrene beads. Following the $\mathrm{Ag}$ nanodisk fabrication a $5 \mathrm{~nm} \mathrm{Si}_{3} \mathrm{~N}_{4}$ layer is deposited on the sample by plasma enhanced chemical vapor deposition. The last step is the formation of $\mathrm{Pd}$ nanoparticles by evaporating a thin non-covering $\mathrm{Pd}$ layer (nominal thickness of $4 \AA$ ) on top of the samples. Because of poor wetting between the $\mathrm{Si}_{3} \mathrm{~N}_{4}$ surface and palladium, nanoparticles are formed. We made samples with three diameters: $64 \mathrm{~nm}, 127 \mathrm{~nm}$, and $167 \mathrm{~nm}$, all having a height of $20 \mathrm{~nm}$. In each case three different samples are made: one reference sample with only $\mathrm{Ag}$ disks and the $\mathrm{Si}_{3} \mathrm{~N}_{4}$ layer, a second reference with a $\mathrm{Si}_{3} \mathrm{~N}_{4}$ layer and $\mathrm{Pd}$, and a full coupled structure of $\mathrm{Ag}$ disks, $\mathrm{Si}_{3} \mathrm{~N}_{4}$ layer, and $\mathrm{Pd}$ particles. The $\mathrm{Si}_{3} \mathrm{~N}_{4}$ layers are included in all samples to assure that its influence on the plasmonic properties as well as the wetting between the $\mathrm{Pd}$ and the sample is the same in all cases. By itself, the $5 \mathrm{~nm} \mathrm{Si}_{3} \mathrm{~N}_{4}$ layer shows no appreciable absorption in the studied range.

Optical extinction and absorption spectra of the samples were measured with a Varian Cary 5000 spectrophotometer. The absorption efficiencies were measured using an integrating sphere accessory, Varian DRA 2500. The samples were mounted in the center of the DRA for transflectance measurements. Scattering spectra are calculated from the difference between extinction and absorption spectra.

\section{INCREASING LIGHT AND PLASMON ABSORPTION IN CATALYTIC METALS}

Silver has, in comparison to many other metals, a relatively low plasma frequency. If one factors in interband absorption that is present below ca. $350 \mathrm{~nm}$, then it is clear why its LSPR of small ca. $20 \mathrm{~nm}$ particles does not shift too far into the UV. In contrast, many other non-noble transition metals, many of them characterized by large losses, have relatively large plasma frequencies and as a result their plasmon resonances for particle sizes in the $20 \mathrm{~nm}$ range are shifted further into the UV than for silver. ${ }^{17}$ From the electromagnetics point of view there is no right or wrong when it comes to the spectrum at which a particle resonates. Indeed, plasmonics in the UV with the poor metals (due to their large losses in the visible), especially aluminum, are quite promising for e.g. enhanced UV SERS with the only limiting factor being available light sources.

SERS is, of course, hardly the only application for plasmonics. A recently opened up field is plasmonenhanced catalysis on metal nanoparticles. ${ }^{21}$ This and other early works focused on noble metals which are 


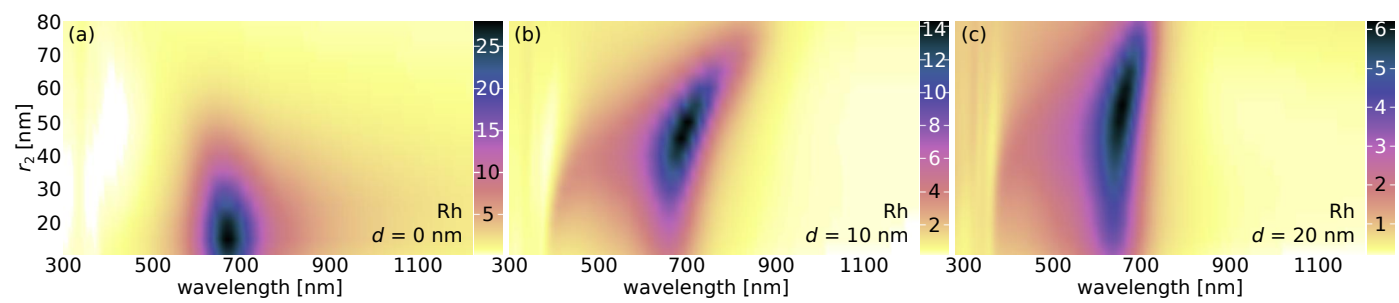

Figure 2. Examples of wavelength-dependent absorption enhancement for rhodium on top of a silver disk of radius $r_{1}=80 \mathrm{~nm}$ and resonator separations: a $d=0 \mathrm{~nm}, \mathbf{b} d=10 \mathrm{~nm}$, and $\mathbf{c} d=20 \mathrm{~nm}$. The maximum enhancement follows the LSPR of the silver disk and within the range of investigated separations moves to larger $r_{2}$ values for increasing $d$. For other metals the qualitative spectral dependence is similar, however, quantitatively enhancement is lower and depends on the metal used. When $r_{1}$ is reduced the LSPR of the Ag disk blueshifts and, consequently, so does the enhancement peak
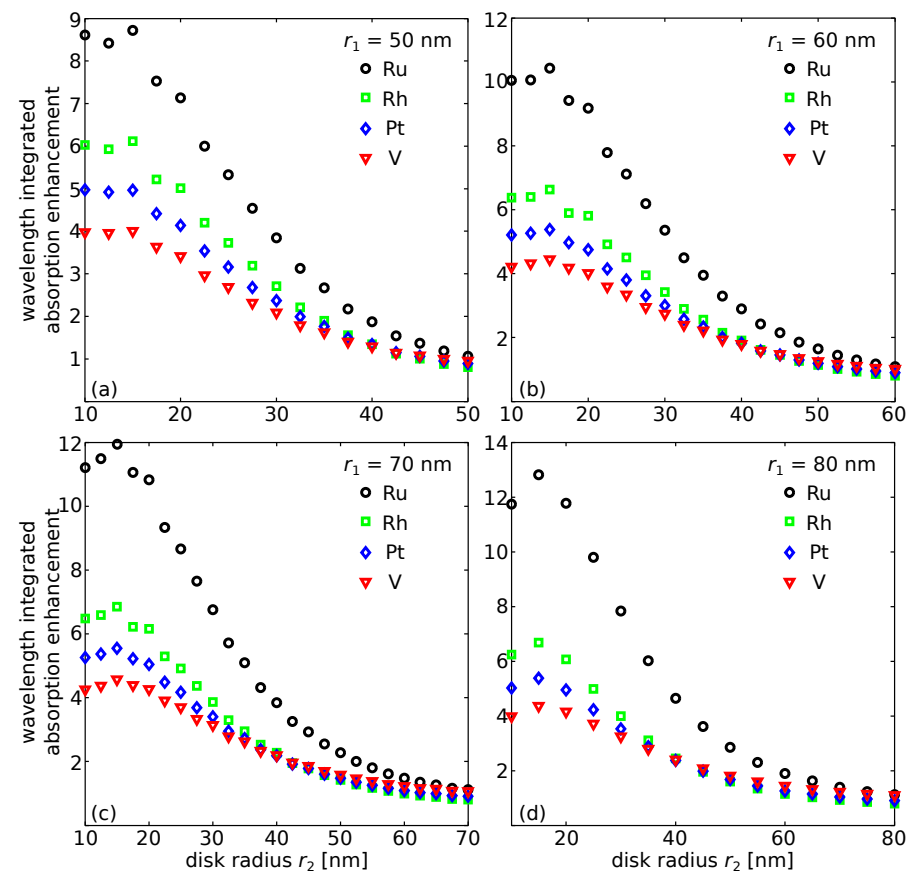

Figure 3. Wavelength-integrated absorption enhancement [Eq. (1)] for touching dimer disks $(\mathrm{d}=0 \mathrm{~nm})$ as function of the top disk radius $\mathrm{r} 2$ for (a) a silver resonator with $\mathrm{r} 1=50 \mathrm{~nm}$, (b) $60 \mathrm{~nm}$, (c) $70 \mathrm{~nm}$, and (d) $80 \mathrm{~nm}$. Despite a large variation in permittivities (up to one order of magnitude) the absorption enhancement of the various metals is relatively similar. Despite an increase of the size of the Ag nanoan-tenna absorption enhancement in $\mathrm{Rh}, \mathrm{Pt}$, and $\mathrm{V}$ remains almost unchanged and only shows an increase in $\mathrm{Ru}$. The enhancement order of $\mathrm{Ru}>\mathrm{Rh}>\mathrm{Pt}>\mathrm{V}$ is directly related to the optical spectra of single disks, which are dictated by their plasma frequencies.

rather poor catalytic metals, ${ }^{16}$ but having a very good plasmonic response makes for excellent proof-of-concept. Other transition metals are excellent catalysts and therefore it is of interest to study their plasmon-assisted photocatalytic properties. A potential and for practical purposes limitless source of energy is the Sun, however, due to the large plasma frequencies of catalytic metals their plasmon response does not overlap the solar spectrum. Thus, shifting their plasmonic activity into the visible spectrum is important from an applications point of view. ${ }^{22,23}$ Here, we show how to do just this using hybridized hetero-metallic nanostructures ${ }^{24}$ which utilize a low-loss nanoantenna to collect incident light and direct it into LSPRs on the catalytic metal nanoparticles. 


\subsection{Coupled nanostructures with large catalytic metal elements}

We begin by coupling relatively large catalytic metal nanoparticles to a silver nanodisk with a variable radius. The best results, i.e. largest absorption enhancement, is achieved for rhodium. Depending on the separation between the two disks, local enhancement varies from ca. $28(d=0 \mathrm{~nm})$ to only 6 for $d=20 \mathrm{~nm}$, see Fig. 2 . The absorption enhancement bands trace the plasmon resonance of the silver disk. When the Ru disk touches the silver disk, large enhancement is only observed for small $r_{2}$. The reason for that is that under touching conditions there is only one plasmon mode which is mostly centered in the silver, while it avoids the Ru part. The other issue is that when the $\mathrm{Ru}$ disk becomes big, it dampens quite severely the plasmon decreasing absorption enhancement. When the Ru disk is moved away from the Ag disk, the enhancement decreases and at the same time the peak moves toward larger $r_{2}$ values and simultaneously decreases in value. Other metals have lower enhancements.

Figure 3 presents total absorption enhancements (wavelength-integrated) for the four metals and for $d=$ $0 \mathrm{~nm}$, since for this separation distance the largest enhancement for $\mathrm{Ru}$ was calculated. Qualitatively all the plots for different silver disk radii look the same with $\mathrm{Ru}$ exhibiting the largest enhancement and the remaining three metals falling behind. The enhancement value order $\mathrm{Ru}>\mathrm{Rh}>\mathrm{Pt}>\mathrm{V}$ and magnitude changes are surprising at first when one considers the dielectric function of the materials, some of which differ by up to an order of magnitude. However, their optical spectra explain the reason for this behavior. ${ }^{23}$ The observed enhancement order is the same as that of the plasma frequencies as observed qualitatively based on absorption efficiencies. Ru has the largest plasma frequency and correspondingly has large absorption enhancement. Following is Rh, then Pt, and finally V.

\subsection{Absorption enhancement in nanometer-sized palladium nanoparticles}

Results of the analysis of absorption enhancement in a palladium particle driven by plasmonic sliver nanodisks of varied diameters are shown in Fig. 4. Absorption is proportional to the square of the electric field in the lossy material, thus absorption in $\mathrm{Pd}$ is maximum when the driving field it is paced in is the largest. This occurs at positions aligned along the polarization which determines the orientation of the induced dipole in the silver nanoantenna. This is observed in Fig. 4a, where we show 3D absorption enhancement maps for 60 and $120 \mathrm{~nm}$ diameter disks - note how the maximum enhancement is aligned along the $x$-axis. The strength of the enhancement depends on the size of the disk, as made evident in Fig. 4b, where we plot minimum, maximum, and average absorption enhancement. They become larger as the radius increases up to $60 \mathrm{~nm}$ when they reach their respective peak values, and then decrease.

In an experiment employing this proposed scheme we expect that positioning of the palladium nanoparticles would be random and, depending on their dimensions (both Ag disk, and catalytic particles), anywhere from the low teens to a few dozen Pd particles will be present near the nanoantenna. The exact number depends on fabrication conditions. In all probability, there will be no control of the $\mathrm{Pd}$ particle positioning, thus we are mostly interested in the average absorption enhancement. In the theoretical analysis this value varies between 7 and 12, depending on the Ag disk size.

This resonant character of absorption enhancement directly stems from the resonant properties of the silver nanoantenna. It is known from optical theory that scattering, while lower at short wavelengths, compared to the scattering particle size, is smaller than absorption, while for longer wavelengths the situation reverses. For a resonance that occurs at high frequencies more energy in the nanoantenna is lost due to absorption, than scattering, yielding less energy to be coupled into the palladium particle. Moreover, as the particle size increases retardation begins to play an increasingly important role lowering the near-field enhancement and, as a consequence, lowering absorption.

The second factor contributing to the absorption enhancement peak is matching the solar spectrum maximum to the size of the driving silver nanodisk. Since absorption is proportional to the square of the electric field, it is optimal to use a nanoantenna which will offer maximum near-field enhancement at the peak of the solar spectrum. This is achieved when extinction peaks around $500 \mathrm{~nm}$. 

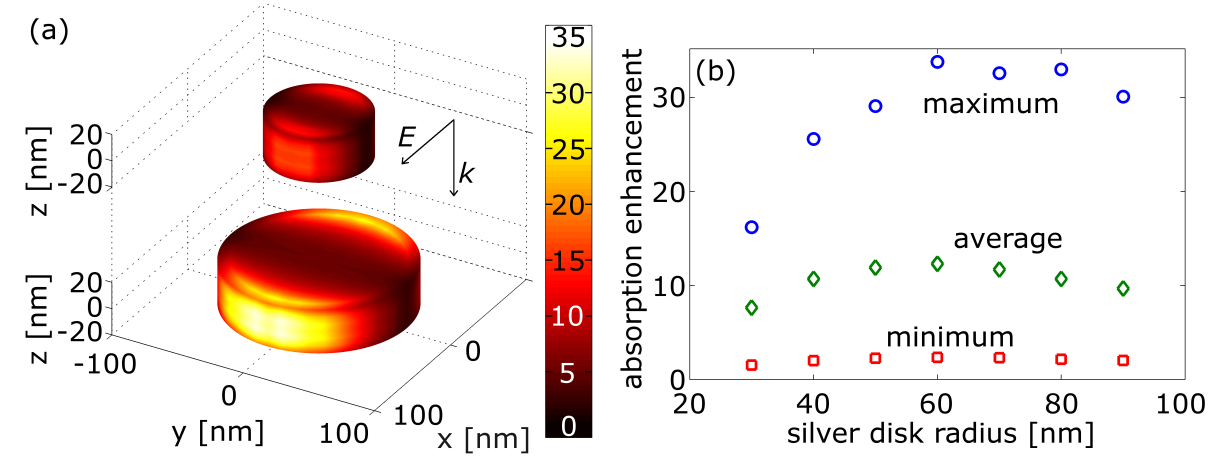

Figure 4. (a) Spatial distribution of absorption enhancement in palladium particles placed near a silver nanodisk 30 (upper disk) and $60 \mathrm{~nm}$ (lower) in radius. The absorption maxima are located along the direction of polarization -16 for $R=30$ and 34 for $60 \mathrm{~nm}$. Minimum absorption enhancement is observed at the sides of the disks at the halfway between the dipole poles. (b) Maximum, average, and minimum absorption enhancement as a function radius exhibit a peak at $R=60 \mathrm{~nm}$ due to retardation limiting the available field enhancement. Randomly deposited Pd particles (only one per silver disk) in an ensemble will experience the average which is 7.6 for $R=30 \mathrm{~nm}$ and reaches 12.3 for $60 \mathrm{~nm}$. Maximum enhancement for $R \geq 60 \mathrm{~nm}$ saturates and then decreases slowly, however, average enhancement drops more quickly because for an increasing radius the relative area with low enhancement is correspondingly larger than for small disks.

\subsection{Absorption enhancement in nanometer-sized palladium nanoparticles}

To test the feasibility of using near-field coupling to increase absorption in Pd nanoparticles we have performed absorption measurements on the samples we fabricated. Absorption efficiency measurements are shown in Fig. 5 for disks 64, 123, and $167 \mathrm{~nm}$ in diameter. In each plot we present three measurements - absorption in an only-Ag array (blue line), in palladium nanoparticles only (red line), and absorption in a coupled Ag-Pd nanostructure (black line). To be able to assert whether coupling increases absorption, we show, using a dashed line, the sum of absorption separately in $\mathrm{Ag}$ and in Pd. For the 123 and $167 \mathrm{~nm}$ disks absorption in the coupled case is clearly larger than then sum. This translates into much larger absorption in Pd. For the smallest disk, $64 \mathrm{~nm}$ in diameter, the sum and the coupled absorption are close in amplitude, but shifted by a few nanometers. In effect the short-wavelength part of the coupled peak is below the sum, while at the long-wavelength side it is larger. This suggests that also in this case absorption in palladium in at least part of the spectrum is enhanced. Exact quantitative measurements of absorption in only part of the coupled nanostructure are at this point not possible, so ideally a potential proxy experiment would be measurement of catalytic reaction rates on such nanostructures with and without light, as well as with Pd nanoparticles coupled to Ag disks and being uncoupled. Attempts at making such experiments are ongoing.

\section{SUMMARY AND CONCLUSIONS}

Using FDTD calculations and experimental measurements we have shown the limits of absorption engineering for a variety of catalytic metals and identified that one important reason behind varied absorption enhancement in different metals is the value of the plasma frequency. The larger it is, the more one can enhance light absorption in a catalytic metal nanostructure by coupling it with a good plasmonic resonator. Wavelengthaveraged absorption enhancements yield values approaching and potentially exceeding one order of magnitude.

We have also investigated absorption enhancement in nanometer-sized nanoparticles made of palladium coupled to a silver nanoantenna (Pd nanoparticle being much smaller than the Ag nanodisk). Average positiondependent absorption enhancement in palladium in the optimum case reaches also one order of magnitude for the case when the Ag nanodisk's LSPR matches the position of the solar maximum. Using these numerical experiments as a basis, we have devised a series of experiments to verify the FDTD calculations. Having 

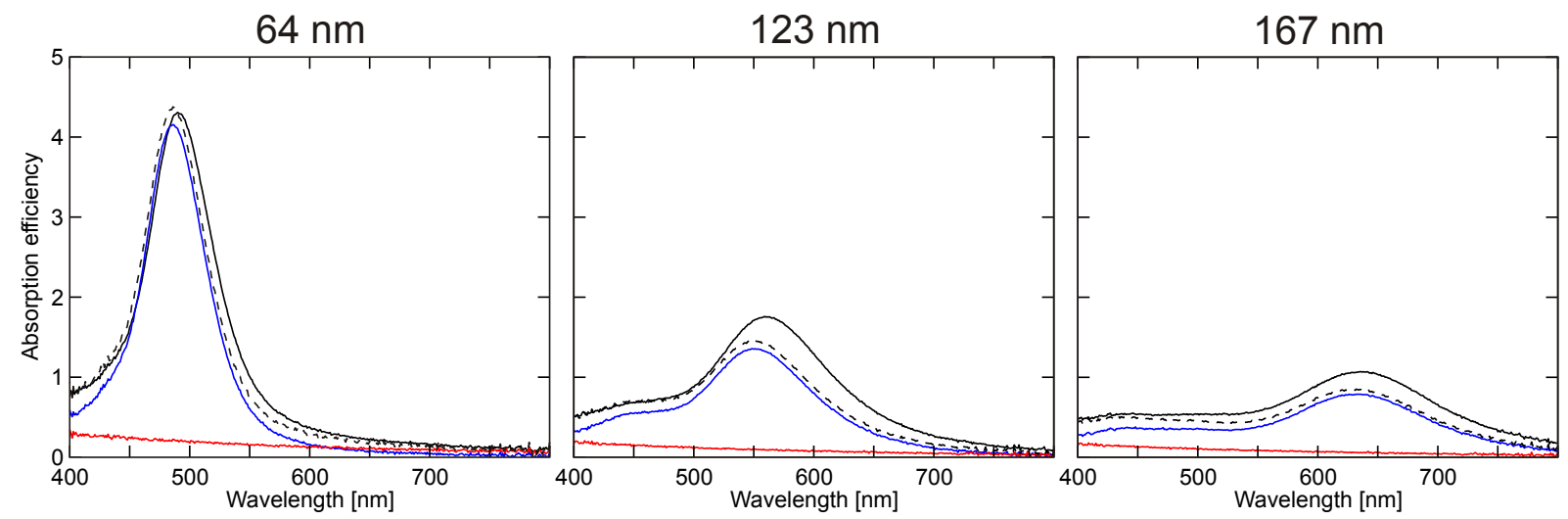

Figure 5. Absorption efficiency of Ag, Pd, and Ag-Pd nanostructures. Ag nanodisks have diameters 64, 123, and $167 \mathrm{~nm}$. The blue line is absorption in $\mathrm{Ag}$ nanodisks alone, the red line absorption in $\mathrm{Pd}$ alone, the dashed line is a sum of absorption in $\mathrm{Ag}$ and $\mathrm{Pd}$, the black line is absorption in an Ag-Pd coupled nanostrufture. Note, that in all cases (least visible for $64 \mathrm{~nm}$ disks) absorption in the coupled structure is considerably larger than than the sum of absorption in $\mathrm{Ag}$ and Pd. Hence, absorption in Pd nanoparticles near the $\mathrm{Ag}$ disks is larger than in $\mathrm{Pd}$ alone. This is due to coupling between the $\mathrm{Ag}$ disks and the $\mathrm{Pd}$ nanoparticles.

fabricated $\mathrm{Ag}-\mathrm{Pd}$ coupled nanostructures, we have measured the absorption efficiency and obtained that absorption in a coupled nanostructure is larger than the sum of absorptions in $\mathrm{Ag}$ and $\mathrm{Pd}$ separately. This is strong evidence of increased light absorption in the catalytic palladium nanoparticles.

\section{ACKNOWLEDGMENTS}

TJA acknowledges support from the Foundation of Polish Science via the project HOMING PLUS/20137/1. This work was also supported by the Swedish Foundation for Strategic Research via the Functional Electromagnetic Metamaterials for Optical Sensing project SSF RMA 11. Simulations were performed in the ICM at the University of Warsaw, Grant No. G55-6.

\section{REFERENCES}

[1] Anker, J. N., Hall, W. P., Lyandres, O., Shah, N. C., Zhao, J., and Van Duyne, R. P., "Biosensing with plasmonic nanosensors," Nature Mater. 7, 442-453 (2008).

[2] McFarland, A. D. and Van Duyne, R. P., "Single silver nanoparticles as real-time optical sensors with zeptomole sensitivity," Nano Lett. 3, 1057-1062 (2003).

[3] Antosiewicz, T. J., Apell, S. P., Claudio, V., and Käll, M., "A simple model for the resonance shift of localized plasmons due to dielectric particle adhesion," Opt. Express 20, 524-533 (2012).

[4] Tang, L., Kocabas, S. E., Latif, S., Okyay, A. K., Ly-Gagnon, D.-S., Saraswat, K. C., and Miller, D. A. B., "Nanometre-scale germanium photodetector enhanced by a near-finfrared dipole antenna," Nature Photon. 2, 226-229 (2008).

[5] Chang, C.-C., Sharma, Y. D., Kim, Y.-S., Bur, J. A., Shenoi, R. V., Krishna, S., Huang, D., and Lin, S.-Y., "A surface plasmon enhanced infrared photodetector based on InAs quantum dots," Nano Lett. 10, 1704-1709 (2010).

[6] Saj, W. M., Antosiewicz, T. J., Pniewski, J., and Szoplik, T., "Energy transport in plasmon waveguides on chain of metal nanoplates," Opto-Electron. Rev. 14, 243-251 (2006).

[7] Ahmadivand, A. and Golmohammadi, S., "Compositional arrangement of rod/shell nanoparticles: an approach to provide efficient plasmon waveguides," Opto-Electron. Rev. 22(2), 101-108 (2014).

[8] Cubukcu, E., Kort, E. A., Crozier, K. B., and Capasso, F., "Plasmonic laser antenna," Appl. Phys. Lett. 89, 093120 (2006). 
[9] Noginov, M. A., Zhu, G., Belgrave, A. M., Bakker, R., Shalaev, V. M., Narimanov, E. E., Stout, S., Herz, E., Suteewong, T., and Wiesner, U., "Demonstration of a spaser-based nanolaser," Nature 460, 1110-1113 (2009).

[10] Ferrara, D. W., Nag, J., MacQuarrie, E. R., Kaye, A. B., and Haglund, R. F., "Plasmonic probe of the semiconductor to metal phase transition in Vanadium Dioxide," Nano Lett. 13, 4169-4175 (2013).

[11] Christopher, P., Xin, H., and Linic, S., "Visible-light-enhanced catalytic oxidation reactions on plasmonic silver nanostructures," Nature Chem. 3, 467-472 (2011).

[12] An, C., Wang, J., Liu, J., Wang, S., and Zhang, Q.-H., "Plasmonic enhancement of photocatalysis over Ag incorporated AgI hollow nanostructures," RSC Adv. 4, 2409-2413 (2014).

[13] Mukherjee, S., Libisch, F., Large, N., Neumann, O., Brown, L. V., Cheng, J., Lassiter, J. B., Carter, E. A., Nordlander, P., and Halas, N. J., "Hot electrons do the impossible: Plasmon-induced dissociation of $\mathrm{H}_{2}$ on Au," Nano Lett. 13, 240-247 (2013).

[14] Kale, M. J., Avanesian, T., and Christopher, P., "Direct photocatalysis by plasmonic nanostructures," ACS Catal. 4, 116-128 (2014).

[15] Linic, S., Christopher, P., Xin, H., and Marimuthu, A., "Catalytic and photocatalytic transformations on metal nanoparticles with targeted geometric and plasmonic properties," Acc. Chem. Res. 46, 1890-1899 (2013).

[16] Hammer, B. and Nørskov, J. K., "Why gold is the noblest of all the metals," Nature 376, 238-240 (1995).

[17] McMahon, J. M., Schatz, G. C., and Gray, S. K., "Plasmonics in the ultraviolet with the poor metals Al, Ga, In, Sn, Tl, Pb, and Bi," Phys. Chem. Chem. Phys. 15, 5415-5423 (2013).

[18] Adibi, P. T. Z., Mazzotta, F., Antosiewicz, T. J., Skoglundh, M., Grönbeck, H., and Langhammer, C., "In situ plasmonic sensing of platinum model catalyst sintering on different oxide supports and in $\mathrm{O}_{2}$ and $\mathrm{NO}_{2}$ atmospheres with different concentrations," ACS Catal. 5, 426-432 (2015).

[19] Palik, E. D., ed., [Handbook of Optical Constants of Solids], vol. 1, Academic Press: New York (1985).

[20] Fredriksson, H., Alaverdyan, Y., Dmitriev, A., Langhammer, C., Sutherland, D. S., Zäch, M., and Kasemo, B., "Hole-mask coloidal lithography," Adv. Mater. 19, 4297-4302 (2007).

[21] Linic, S., Christopher, P., and Ingham, D. B., "Pasmonic-metal nanostructures for efficient conversion of solar to chemical energy," Nature Mater. 10, 911-921 (2011).

[22] Antosiewicz, T. J., Apell, S. P., Wadell, C., and Langhammer, C., "Absorption enhancement in lossy transition metal elements of plasmonic nanosandwiches," J. Phys. Chem. C 116, 20522-20529 (2012).

[23] Antosiewicz, T. J. and Apell, S. P., "Optical enhancement of plasmonic activity of catalytic metal nanoparticles," RSC Adv. 5, 6378-6384 (2015).

[24] Wadell, C., Antosiewicz, T. J., and Langhammer, C., "Optical absorption engineering in stacked plasmonic au-sio 2 -pd nanoantennas," Nano Lett. 12, 4784-4790 (2012). 